\title{
Séance d'apprentissage du raisonnement clinique (ARC) à distance
}

\section{Distance learning clinical reasoning sessions}

\author{
Valérie MASSART ${ }^{*}$, Laetitia BURET, Didier GIET, Jean-Luc BELCHE, et Valérie DORY \\ Département de médecine générale, Faculté de médecine, Université de Liège, Liège, Belgique
}

Manuscrit reçu le 22 septembre 2020 ; accepté pour publication le 19 novembre 2020

\section{Contexte}

Le cursus médical à l'Université de Liège (ULiège - Belgique) dure six années, divisées en une phase préclinique (2,5 années), et une phase clinique (3,5 années), comprenant des cours et des stages (stages courts de la troisième à la cinquième année, la sixième étant consacrée entièrement aux stages). Des séances d'apprentissage du raisonnement clinique (ARC) sont organisées en troisième, quatrième et cinquième années, et visent la mise en pratique des enseignements théoriques. Le Département de médecine générale organise 12 séances d'ARC [1]: trois en troisième année, six en quatrième année, trois en cinquième année. Le confinement décidé en réponse à la pandémie COVID-19 a poussé l'université à transformer les activités pédagogiques vers des modalités à distance. Au moment du confinement, il restait trois séances d'ARC à assurer pour l'année académique en cours.

\section{Problématique}

Le raisonnement clinique (RC) correspond aux processus de pensée qui permettent au clinicien de prendre les décisions sur les actions les plus appropriées dans un contexte spécifique de résolution de problème de santé $[1,2]$.

Les séances spécifiques d'ARC, développées par Chamberland [2], devaient impliquer explicitement les étudiants dans une démarche clinique et favoriser chez les étudiants en stage, une construction et une ré-organisation actives de connaissances cliniques $[1,2]$. Selon la méthode originale, une situation clinique sous forme de consultation d'un malade chez un médecin est présentée à des petits groupes de six à huit étudiants, sur leur lieu de stage,

\footnotetext{
*Correspondance et offprints : Valérie MASSART, Département médecine générale, Faculté de médecine, Université de Liège, Domaine du Sart Tilman (Bât. B23), 4000 Liège, Belgique. Mailto: V.Massart@chu.ulg.ac.be.
}

durant 60 à 90 minutes. Les étudiants abordent un problème et une entité pathologique définis. Ils élaborent à voix haute les étapes intermédiaires du $\mathrm{RC}$, aidés par un tuteur [2].

L'ULiège organise également des séances d'ARC d'une durée de $1 \mathrm{~h}$, par groupes de dix étudiants (soit 20 groupes pour chaque cohorte de 200 étudiants environ), selon la même méthode mais dans des locaux neutres. La contextualisation se fait essentiellement au moyen de la présentation du cas.

$\mathrm{Au}$ vu du contexte pandémique, le Département de médecine générale a envisagé la transformation des séances d'ARC restantes en formation à distance, à condition que les étudiants soient familiers de la méthode. Deux séances concernant des étudiants qui n'y avaient jamais participé ont ainsi été annulées et la dernière transformée en formation à distance.

\section{Ce qui a été fait}

Douze tuteurs ont répondu être disponibles et enthousiastes pour tenter l'expérience d'animer une séance dans une classe virtuelle. Le scénario était bien connu des tuteurs et concernait le saignement gynécologique (spotting). Une chargée de communication a assuré la formation technique accélérée des tuteurs sur l'utilisation de l'outil en ligne institutionnel (Blackboard Collaborate), d'abord par un contact téléphonique puis en les invitant à se connecter à la plateforme en guise d'exercice. Une secrétaire a organisé les groupes d'étudiants et les a informés des modalités pratiques de la réunion virtuelle. La séance d'ARC à distance a ainsi été préparée en quatre jours par deux personnes. Dès le lendemain, $46 \%$ des étudiants $(n=97)$ et 11 des 12 tuteurs ont répondu à des questionnaires en ligne spécifiques, comportant respectivement des questions ouvertes (tableau I), des questions à réponse selon une échelle de Likert à 5 points (tableau II) et des questions fermées visant à comparer (au regard d'une plus-value, d'une valeur identique ou d'une moinsvalue) les séances en présence et à distance (tableau III). 
Tableau I. Questionnaire d'avis sur les séances d'apprentissage du raisonnement clinique (ARC) à distance.

\section{Questions ouvertes posées aux étudiants}

Globalement, quels ont été les avantages d'une séance d'ARC à distance?

Globalement, quelles ont été les difficultés, désavantages d'une séance d'ARC à distance?

\section{Questions ouvertes posées aux tuteurs}

Si vous avez vécu deux séances, cela s'est-il passé de façon similaire? Quelles ont été les différences/difficultés?

Avez-vous rencontré des difficultés dans l'animation d'une séance d'ARC à distance? Si oui, lesquelles?

Que pensez-vous de l'exercice du raisonnement clinique par chaque étudiant durant la séance à distance? (Tous les étudiants peuvent s'exercer à la démarche du RC en tout ou en partie?)

Tableau II. Questionnaire d'opinion relatif aux séances d'apprentissage du raisonnement clinique (ARC) à distance (réponses selon une échelle de type Likert).

\begin{tabular}{|c|c|c|c|c|c|c|}
\hline & \multicolumn{3}{|c|}{ Étudiants $(n=97)$} & \multicolumn{3}{|c|}{ Tuteurs $(n=11)$} \\
\hline & Accord $(\%)$ & Neutre (\%) & Désaccord (\%) & Accord $(\%)$ & Neutre $(\%)$ & Désaccord (\%) \\
\hline La séance d'ARC à distance s'est & \multicolumn{3}{|c|}{$n=93$} & \multicolumn{3}{|c|}{$n=10$} \\
\hline globalement bien déroulée & 98 & 0 & 2 & 100 & 0 & 0 \\
\hline Les conditions d'ARC à distance & \multicolumn{3}{|c|}{$n=96$} & \multicolumn{3}{|c|}{$n=10$} \\
\hline $\begin{array}{l}\text { m'ont permis de prendre la parole / } \\
\text { ont permis la prise de parole de } \\
\text { tous les étudiants présents }\end{array}$ & 87 & 6 & 6 & 60 & 0 & 40 \\
\hline La séance d'ARC à distance était & \multicolumn{3}{|c|}{$n=97$} & \multicolumn{3}{|c|}{$n=10$} \\
\hline $\begin{array}{l}\text { agréable / J'ai trouvé l'animation } \\
\text { de l'ARC à distance agréable }\end{array}$ & 89 & 6 & 4 & 90 & 0 & 10 \\
\hline La séance d'ARC à distance & \multicolumn{3}{|c|}{$n=95$} & \multicolumn{3}{|c|}{$n=10$} \\
\hline $\begin{array}{l}\text { a stimulé mon raisonnement } \\
\text { clinique / La séance d'ARC } \\
\text { à distance a été efficace pour } \\
\text { stimuler le raisonnement } \\
\text { clinique des étudiants présents }\end{array}$ & 78 & 17 & 5 & 90 & 0 & 10 \\
\hline $\begin{array}{l}\text { Il y a eu des difficultés techniques } \\
\text { pendant l'ARC à distance }\end{array}$ & 38 & $\begin{array}{l}n=96 \\
18\end{array}$ & 45 & 30 & $\begin{array}{l}n=10 \\
0\end{array}$ & 70 \\
\hline $\begin{array}{l}\text { J'ai trouvé l'animation de l'ARC } \\
\text { à distance aisée }\end{array}$ & / & l & / & \multicolumn{3}{|c|}{$n=10$} \\
\hline $\begin{array}{l}\text { J'accepterais d'animer à nouveau } \\
\text { un ARC à distance }\end{array}$ & / & / & / & \multicolumn{3}{|c|}{$n=10$} \\
\hline Tous les ARC de médecine générale & & $n=95$ & & \multicolumn{3}{|c|}{$n=10$} \\
\hline $\begin{array}{l}\text { à l'avenir, même après la crise sanitaire, } \\
\text { pourraient être organisés à distance }\end{array}$ & 65 & 16 & 19 & 70 & 0 & 30 \\
\hline $\begin{array}{l}\text { Tous les ARC de médecine générale } \\
\text { à l'avenir, même après la crise } \\
\text { sanitaire, devraient être organisés } \\
\text { à distance }\end{array}$ & 36 & $\begin{array}{l}n=96 \\
23\end{array}$ & 41 & 20 & $\begin{array}{l}n=10 \\
0\end{array}$ & 80 \\
\hline
\end{tabular}

\section{Ce qui a été observé}

Les réponses aux questions fermées sont présentées dans les tableaux II et III.

La quasi-totalité des étudiants rapportent des avis positifs. Lorsqu'ils développent leur avis, les étudiants relèvent le gain de temps comme bénéfice premier (pas de déplacements, pas d'attente avant l'ARC, remise au travail dès l'ARC terminé). Certains évoquent aussi le temps gagné pour leur tuteur médecin généraliste qui ne doit pas se déplacer, trouver une place de parking, etc. ce qui, selon les étudiants, peut lui permettre de consacrer plus de temps à ses patients. Beaucoup d'étudiants évoquent spontanément la liberté de parole que permet le système à distance: «on ose davantage prendre la parole», «on ne voit pas tout le monde» (note des auteurs : pas d'exposition des corps, donc on ose plus), «cela peut permettre à des étudiants plus timides de prendre la parole». Plusieurs parlent de l'écoute et du respect mutuels de la parole des autres. 
Tableau III. Appréciation comparative des séances d'apprentissage du raisonnement clinique (ARC) en présence ou à distance, du point de vue des étudiants et des tuteurs.

\begin{tabular}{|c|c|c|c|c|c|c|c|c|}
\hline & \multicolumn{2}{|c|}{$\begin{array}{l}\text { Nombre de } \\
\text { répondants }\end{array}$} & \multicolumn{2}{|c|}{$\begin{array}{c}\text { Plus-value de } \\
\text { l'ARC à distance (en \%) }\end{array}$} & \multicolumn{2}{|c|}{$\begin{array}{c}\text { Valeur identique } \\
\text { de l'ARC en présence } \\
\text { ou à distance (en \%) }\end{array}$} & \multicolumn{2}{|c|}{$\begin{array}{l}\text { Moins-value de l'ARC } \\
\text { à distance (en \%) }\end{array}$} \\
\hline & Étudiants & Tuteurs & Étudiants & Tuteurs & Étudiants & Tuteurs & Étudiants & Tuteurs \\
\hline $\begin{array}{l}\text { Au niveau de vos apprentissages } \\
\text { du raisonnement clinique }\end{array}$ & 94 & 6 & 33 & 40 & 67 & 20 & 0 & 40 \\
\hline $\begin{array}{l}\text { En matière de communication, } \\
\text { d'échanges, prises de parole }\end{array}$ & 95 & 6 & 60 & 20 & 40 & 20 & 0 & 60 \\
\hline Temps pour le tuteur & & 6 & & 43 & & 43 & & 14 \\
\hline
\end{tabular}

Tableau IV. Points forts et points faibles des séances d'apprentissage du raisonnement clinique (ARC) en présence ou à distance.

\begin{tabular}{|c|c|c|}
\hline & Séances d'ARC à distance & Séances d'ARC en présence \\
\hline Gains de déplacement/temps & +++ & - \\
\hline Facilité de prise de parole & ++ & $+/-$ \\
\hline Structuration de la prise de parole & ++ & + \\
\hline Connexion/technique & $-/-$ & +++ \\
\hline Contact social & + & ++ \\
\hline
\end{tabular}

D'ailleurs, $60 \%$ des étudiants trouvent que la séance à distance revêt une plus-value en matière de communication, d'échanges et de prises de parole. La séance avance plus vite, est plus structurée et plus efficace qu'une séance classique. Certains étudiants disent être plus concentrés et mieux réfléchir à distance. Les cours sont à portée de main, les étudiants peuvent s'y plonger dès la séance terminée. La participation à distance en petits groupes a permis à certains de ressentir à nouveau un lien avec l'université et un lien social : «cela m'a fait du bien d'avoir une activité interactive après cinq semaines de confinement».

Les inconvénients sont beaucoup moins nombreux que les avantages. Les plus importants concernent les problèmes techniques (coupures d'Internet, problèmes de son et de caméra), éprouvés par certains groupes. Certains étudiants soulignent le manque d'interactions spontanées, les difficultés liées à la prise de parole simultanée d'étudiants dans le groupe à distance, la difficulté à rester concentrés, ou parfois le manque de contacts sociaux en présence.

Les tuteurs rapportent également très majoritairement une expérience positive. Qualitativement, ils évoquent l'impact des difficultés d'ordre technique sur l'animation. Certains étudiants n'ont pas allumé leur camera, ce qui ne facilite pas l'interaction avec le tuteur et le groupe. Cela empêche aussi le tuteur de prendre en compte le comportement non verbal et de visualiser l'activité des étudiants. Les tuteurs qualifient ou considèrent l'exercice du RC chez les étudiants comme bon et d'autres comme plus difficile à exercer. Au minimum, l'étudiant doit participer à une séance pour exercer son RC.
Après la crise sanitaire, les deux tiers des étudiants et des tuteurs affirment que les séances à distance pourraient être à nouveau organisées, mais près de la moitié des étudiants et huit tuteurs pensent que les séances à distance ne devraient pas remplacer totalement les séances en présence.

\section{Points forts}

L'organisation de séances d'ARC à distance a permis aux étudiants, dans ce contexte exceptionnel, de poursuivre la mise en pratique de leur raisonnement clinique dont on sait que l'apprentissage est important [1], et de préserver une modalité interactive dans une période d'isolement pour les étudiants.

\section{Points faibles}

Les difficultés et obstacles principaux du dispositif à distance sont liés au matériel informatique des étudiants et à la connexion internet. Le risque est de renforcer les inégalités entre étudiants. Le département et les tuteurs doivent aussi mettre en place des règles de bon fonctionnement (règles de prise de parole, vidéo ouverte...) pour permettre l'interaction sociale minimale pour les interactions et l'animation [3].

Le tableau IV dresse un inventaire comparatif des points forts et des points faibles de chaque modalité.

$\mathrm{Si}$ ces résultats préliminaires sont encourageants, la satisfaction des étudiants ne nous permet pas de conclure sur leurs apprentissages en tant que tels. La perception positive du dispositif pourrait être liée à la reprise de 
contact avec les pairs et l'université en général qu'il a permis, peu d'activités synchrones ayant été organisées en remplacement des cours en présence mais l'avis positif rend cette hypothèse moins plausible.

Toutefois, les avis des tuteurs sont plus mitigés que ceux des étudiants : $40 \%$ d'entre eux trouvent une plusvalue, $40 \%$ une moins-value par rapport aux séances en présence. Il est possible que certains aient été plus stressés que les étudiants par la survenue d'incidents techniques ou l'anticipation d'avoir à gérer cette situation. Leur moindre satisfaction de la communication pourrait résulter des incidents critiques mais peut-être également de leur moindre maîtrise du flux des discussions dans un contexte à distance, qui horizontalise les relations. Cela pourrait aussi être dû à la profession des tuteurs, qui sont d'abord tous des médecins généralistes. La distance avec les étudiants est contraire au métier de contact qu'ils incarnent.

Il se pourrait enfin qu'ils soient plus conscients que les étudiants des limites du dispositif, notamment en termes de contextualisation. Le déficit de contextualisation déjà noté pour nos séances en présence (locaux universitaires, cadre non médical) s'est trouvé accentué à distance, les étudiants se retrouvant isolés, à leur domicile pour la plupart. Le sentiment de devenir médecin pourrait ainsi s'amenuiser.

Néanmoins, ces premiers résultats et la poursuite de la pandémie nous font envisager de poursuivre l'expérience. En supprimant le temps de déplacement des tuteurs, nous pourrions en recruter davantage, réduire ainsi la taille des groupes et donc favoriser les interactions. On peut également supposer que l'expérience accroîtra l'aisance des tuteurs et des étudiants. Nous pourrons également prévoir une évaluation plus approfondie des effets des séances à distance sur l'apprentissage des étudiants.

\section{Conclusion}

L'organisation de séances d'ARC à distance semble permettre non seulement un gain de temps mais, également, une prise de parole plus aisée des étudiants. Elle implique cependant des conditions matérielles et organisationnelles minimales.

Cette méthode, qui s'est avérée être une solution adéquate de dépannage, pourrait devenir une modalité à poursuivre, en alternance avec les dispositifs en présence, lors de la sortie du confinement, avantages et inconvénients se contrebalançant.

\section{Références}

1. Nendaz M, Charlin B, Leblanc V, Bordage G. Le raisonnement clinique: données issues de la recherche et implications pour l'enseignement. Pédagogie Médicale 2005;6:235-54.

2. Chamberland M. Un exemple d'activité pédagogique contextualisée adaptée aux stages cliniques en médecine. Ann Med Interne 1998;149:479-84.

3. Winterburn S, Rosenbaum M. Top tips for delivering communication skills teaching online. Salisbury (UK): EACH International Association for Communication in Healthcare. 2020 [On-line] Disponible sur : https://www.each.eu/resour ces/covid-19/.

Citation de l'article : Massart V., Buret L., Giet D., Belche J.-L., Dory V. Séance d'apprentissage du raisonnement clinique (ARC) à distance. Pédagogie Médicale 2020:21;223-226 